\title{
Systematic assessment of health care perception in adults with congenital heart disease in Germany
}

\author{
Rhoia Neidenbach $^{1,2}$, Stephan Achenbach ${ }^{3}$, Caroline Andonian ${ }^{1}$, Ulrike M. M. Bauer ${ }^{4}$, Peter Ewert ${ }^{1}$, \\ Sebastian Freilinger ${ }^{1}$, Ulrike Gundlach ${ }^{3}$, Ann-Sophie Kaemmerer ${ }^{1}$, Nicole Nagdyman ${ }^{1}$, Kathrin Nebel ${ }^{1}$, \\ Renate Oberhoffer ${ }^{1,5}$, Lars Pieper ${ }^{6}$, Wibke Reinhard $^{7}$, Linda Sanftenberg ${ }^{8}$, Fabian von Scheidt ${ }^{1}$, \\ Jörg Schelling $^{9}$, Lavinia Seidel ${ }^{1}$, Michael Weyand ${ }^{10}$, Harald Kaemmerer ${ }^{1}$
}

${ }^{1}$ Department of Congenital Heart Disease and Pediatric Cardiology, German Heart Center Munich, Technical University Munich, Germany; ${ }^{2}$ Department of Sports Medicine, Exercise Physiology and Prevention, Institute of Sport Science, University of Vienna, Austria; ${ }^{3}$ Department of Cardiology, Friedrich-Alexander-University Erlangen-Nürnberg (FAU), Germany; ${ }^{4}$ Competence Network for Congenital Heart Defects, National Register for Congenital Heart Defects, Berlin, Germany; ${ }^{5}$ Department of Preventive Pediatrics, Department of Sport and Health Sciences, Technical University of Munich, Germany; ${ }^{6}$ Department of Behavioral Epidemiology, Technical University of Dresden, Germany; ${ }^{7}$ Department of Cardiovascular Diseases, German Heart Center Munich, Technical University Munich, Germany; ${ }^{8}$ Institute of General Practice, LudwigMaximilians-University Munich, Germany; ${ }^{9}$ Ludwig-Maximilians-University Munich, Germany; ${ }^{10}$ Department of Cardiac Surgery, FriedrichAlexander-University Erlangen-Nürnberg (FAU), Erlangen, Germany

Contributions: (I) Conception and design: All authors; (II) Administrative support: All authors; (III) Provision of study materials or patients: All authors; (IV) Collection and assembly of data: All authors; (V) Data analysis and interpretation: All authors; (VI) Manuscript writing: All authors; (VII) Final approval of manuscript: All authors.

Correspondence to: Rhoia Neidenbach, PhD. Department of Congenital Heart Disease and Pediatric Cardiology, German Heart Center Munich, Technical University Munich, Germany. Lazaresttstr. 36, 80636 Munich, Germany. Email: rhoia.neidenbach@tum.de.

Background: The number of adults with congenital heart disease (ACHD) is steadily rising due to increased survival rate and improved medical resources. Accordingly, more than 330,000 ACHD are currently living in Germany. Almost all of them require lifelong specialized medical follow-up for their chronic heart disease, often accompanied by residua, sequelae, or comorbidities. Primary care physicians (PCPs) are a crucial factor in directing patients to ACHD specialists or specialized institutions, but despite all efforts, the number of ACHD under specialized care is low, the lost-to-follow-up rate is high, and the morbidity and mortality is substantial. The present cross-sectional study was designed to systematically characterize the health care of ACHD from a patient perspective, including (I) use of medical care by ACHD, (II) medical counselling needs, and (III) perceived satisfaction with health care.

Methods: The German-wide analysis was based on a 25 -item questionnaire designed to address different aspects of medical status and health issues of ACHD from their own perspective, performed between May of 2017 and July of 2020.

Results: A total of 4,008 ACHD (52\% female; mean age $41.9 \pm 17.2$ years) completed the questionnaire. The majority of ACHD $(3,524,87.9 \%)$ reported, that they consulted their PCP for non-cardiac health problems, and $49.7 \%(n=1,991)$ consulted their PCP also for medical problems associated with the underlying CHD. Almost all ACHD reported a need for medical advice concerning exercise capacity and daily life activities, occupational skills, pregnancy, rehabilitation, genetic counselling, insurance, and retirement. A total of 1,840 (45.9\%) patients were not aware of the existence of certified ACHD specialists or centers. Moreover, 2,552 $(67.6 \%)$ of those surveyed were uninformed about patient organizations for ACHD.

Conclusions: The present study demonstrates that ACHD are largely uninformed about the ACHD care structures available nationwide, although the patients have a great need for specialized follow-up, advice, and care.

Keywords: Adults with congenital heart disease (ACHD); medical health supply; primary health care; general practitioners; primary care physicians (PCPs); congenital heart disease (CHD) 
Submitted Sep 26, 2020. Accepted for publication Dec 18, 2020.

doi: $10.21037 / \mathrm{cdt}-20-825$

View this article at: http://dx.doi.org/10.21037/cdt-20-825

\section{Introduction}

Congenital heart defects (CHD) are defined as gross structural abnormalities of the heart or intrathoracic great vessels that are functionally significant or of potential functional significance. They are the most common isolated organ anomalies, accounting for about $30 \%$ of all congenital malformations in Europe $(1,2)$. Prevalence estimations of CHD range from 5.2 to 10.7 per 1,000 live births (3-5). The number of children born with CHD per year is about 6,500 in Germany, 108,000 in Europe, and about 1.35 million worldwide (6-8). While in the 1930 s, $80 \%$ of children with relevant CHD died in the first years of life, nowadays, more than $90 \%$ of CHD patients reach adulthood due to remarkable advances in cardiac surgery, paediatric cardiology, intensive care, and pharmacotherapy $(6,9,10)$. It is estimated that, today, the number of adults living with CHD totals more than 330,000 in Germany, 2.3 million in Europe, and 12 to 34 million worldwide (11-13); the population of "adults with congenital heart defects" (ACHD) now exceeds the number of children with CHD.

Despite the enormous reduction in mortality, all patients with CHD still have a chronic heart condition. In almost all ACHD, anatomic or hemodynamic residua and sequelae exist from the medical intervention, and cardiac and/or non-cardiac comorbidities can develop (14-16). The most common cardiac comorbidities include heart failure, arrhythmia and pulmonary hypertension, aortopathy, and endocarditis, while non-cardiac comorbidities encompass metabolic disorders and endocrinological, hepatic, nephrological, and neurological disease (17-19). Moreover, ACHD frequently experience psychological limitations such as depression, anxiety, or posttraumatic stress disorders (20-22).

In order to ensure adequate care for ACHD, a three-stage care model has been developed in Germany, including an ACHD-specific certification for cardiologists or pediatric cardiologists as well as for specialized centers (23). Within this system, basic medical care should be provided by primary care physicians (PCP), including general physicians, family doctors, internists, and pediatricians who participate in general medical care. These physicians have a central role to play in CHD care, through referral of patients to ACHD-specialized institutions. The second level of care includes, at present, 11 regional ACHD hospitals and practices, in which resident adult or pediatric cardiologists offer services for ACHD as well as close-tohome treatment by ACHD-certified cardiologists. The third level currently includes 20 national ACHD centers for tertiary care, which also provide cardiac surgical care and have specialized outpatient departments (e.g., for Marfan syndrome, pulmonary hypertension, pregnancy, and genetic counselling) (24-28). A total of about 350 cardiologists and pediatric cardiologists are accredited as ACHD specialists in Germany.

The aim of this study was to evaluate the current ACHD health care system in Germany from the patient perspective, in order to clarify to what extent ACHD know about and frequent these care structures, which ACHD medical counselling needs are being met, and whether ACHD were satisfied with their perceived health care. We present the following article in accordance with the SURGE reporting checklist (available at http://dx.doi.org/10.21037/cdt-20-825).

\section{Methods}

\section{Study cobort}

This cross-sectional study was a sub-analysis of a nationwide "VEmaH-registry" (www.vemah.info), the first large-scale attempt to analyze the real-world health care status of ACHD in Germany from the patients' perspective. The analysis was performed between May of 2017 and July of 2020.

The study employed a questionnaire-based survey that was initiated by the Department of Congenital Heart Disease of the German Heart Center Munich, Technical University Munich. Inclusion criteria included the presence of a confirmed CHD, a minimum age of 18 years, and a written declaration of consent. Exclusion criteria were lack of cognitive competence to consent to research and refusal to consent. The study was conducted in accordance with the Declaration of Helsinki (as revised in 2013).

The definition of a PCP in the current survey is based on legal requirements. Accordingly, in Germany, primary medical care is provided by all doctors who participate in general medical care. This includes "general practitioners", physicians with basic medical training who are usually the 
Table 1 Socio-demographic variables of the responding adults with congenital heart disease $(\mathrm{N}=4,008)$

\begin{tabular}{lc}
\hline Patient information & Values \\
\hline Age in years, mean $\pm \mathrm{SD} /$ & $41.9 \pm 17.2 / 38[18-97]$ \\
median [range] & \\
Degree of disability, mean $\pm \mathrm{SD} /$ & $35.8 \pm 32.5 / 30[0-100]$ \\
median [range] & \\
Sex, $\mathrm{n}(\%)$ & $1,807(45.1)$ \\
Male & $2,084(52.0)$ \\
Female & $117(2.9)$ \\
Missing data & \\
Place of residence, $\mathrm{n}(\%)$ & $774(19.3)$ \\
Big city & $589(14.7)$ \\
City & $923(23.0)$ \\
Small town & $1,562(39.0)$ \\
Rural community & $160(4.0)$ \\
Missing data & \\
Status of insurance, $\mathrm{n}(\%)$ & $219(5.5$ \\
By law & $3,700(92.3)$ \\
Private & \\
None & \\
Missing data & \\
\hline Big city 100.2$)$ \\
\hline
\end{tabular}

Big city >100,000 inhabitants; city $>20,000-100,000$ inhabitants; small town $>5,000-20,000$ inhabitants; rural community $<5,000$ inhabitants. $\mathrm{N}$, total number; $\mathrm{n}$, absolute number; SD, standard deviation.

first point of contact for the patient if a medical problem arises, and others who participate in primary medical care, including "specialists in general medicine", "specialists in internal medicine and general medicine", and "specialists in internal medicine".

\section{Data collection procedure and measurements}

Several ACHD specialists, epidemiologists, PCPs, and psychologists from different federal states in Germany jointly developed the questionnaire to describe the perceived health care in a large ACHD cohort. The questionnaire comprised 25 questions regarding patientreported diagnosis, residua, sequelae, and cardiac or noncardiac comorbidities. Further included were questions concerning health care structures known and used by
ACHD, and questions asking ACHD to describe their counselling needs and their perceived satisfaction with their current health care.

Data collection was carried out using a questionnaire addressed to ACHD ( $\geq 18$ years) presenting at the Department of Congenital Heart Disease and Pediatric Cardiology of the German Heart Center Munich and the Department of Cardiology of the University of Erlangen. Additionally, the insurance company "AOK Bayern" provided the questionnaires to their policyholders with CHD in Bavaria, and the "National Register for Congenital Heart Defects" in Berlin, Germany, invited its members to participate in the study online. All data collection was conducted with the master-approval of the Ethics Committee of the Technical University of Munich, conferred on April 5, 2017 (157/16 S).

\section{Statistical analysis}

Descriptive statistical analysis was performed using IBM SPSS Statistics 25.0 (IBM Inc., Armonk, NY, USA) to characterize the study population. Continuous data were expressed as arithmetic mean \pm standard deviation (SD), and nominal and categorical variables were expressed as absolute numbers or percentages. The numbers of valid answers given to some questions differed from the total number of the included study participants. This was due to the presence of multiple answers in some cases and missing data in others.

\section{Results}

Questionnaires filled in by 4,008 ACHD (52\% female) were included in the final data analysis. The estimated response rate could not be determined because the majority of the questionnaires were sent by mail or ACHD were invited to participate online; it could not be determined whether the recipients actually received the mailing or opened the online invitation to participate.

The mean age of the included ACHD was $41.9 \pm 17.2$ (range, 18-97; median 38) with a mean degree of disability of $35.8 \pm 32.5$ (range, $0-100$; median 30 ). All sociodemographic characteristics of the surveyed patients are summarized in Table 1 .

The most frequent patient-reported CHDs were aortic valve disease, atrial septal defect, transposition of the great arteries, tetralogy of Fallot, and aortic coarctation (Table 2). According to their own statements, 396 (9.9\%) patients 
Table 2 Patient-reported type of congenital heart defect $(\mathrm{N}=4,008)$

\begin{tabular}{lc}
\hline Type of CHD (multiple answers possible) & $\mathrm{n}(\%)$ \\
\hline Aortic valve stenosis/insufficiency & $718(17.9)$ \\
Atrial septal defect & $544(13.6)$ \\
Ventricular septal defect & $508(12.7)$ \\
Transposition of the great arteries & $349(8.7)$ \\
Tetralogy of Fallot & $340(8.5)$ \\
Aortic coarctation & $327(8.2)$ \\
Pulmonary valve stenosis/insufficiency & $279(7.0)$ \\
Atrioventricular septal defect & $86(2.1)$ \\
Persistent ductus arteriosus & $73(1.8)$ \\
Univentricular heart & $55(1.4)$ \\
Hypoplastic left heart syndrome & $15(0.4)$ \\
Other CHD & $681(17.0)$ \\
Missing data & $33(0.7)$ \\
\hline
\end{tabular}

$\mathrm{CHD}$, congenital heart disease; $\mathrm{N}$, total number; $\mathrm{n}$, absolute number.

Table 3 Patient-reported cardiac and non-cardiac comorbidities ( $\mathrm{N}=3,985$, multiple answers possible)

\begin{tabular}{lc}
\hline Comorbidities & $\mathrm{N}(\%)$ \\
\hline Cardiac arrhythmias & $1,178(29.6)$ \\
Heart failure & $514(12.9)$ \\
Mental or intellectual impairment & $307(7.7)$ \\
Coagulation disorders & $251(6.3)$ \\
Neurological complications & $160(4.0)$ \\
Coronary artery disease & $154(3.9)$ \\
Pulmonary (arterial) hypertension & $148(3.7)$ \\
Thromboembolism & $146(3.6)$ \\
Hematologic disorders & $109(2.7)$ \\
Infective endocarditis & $57(1.4)$ \\
Unknown & $210(5.3)$ \\
None & $1,600(39.9)$ \\
\hline
\end{tabular}

$\mathrm{N}$, total number; $\mathrm{n}$, absolute number.

listed a combination of more than one defect.

Among 3,985 (99.4\%) responses regarding the presence of cardiac and non-cardiac comorbidities, $60 \%(n=2,408)$ of the ACHD reported having relevant comorbidities. Most of these indicated cardiac arrhythmia, heart failure, mental or intellectual impairment, coagulation disorders, and pulmonary (arterial) hypertension (Table 3).

\section{Patient-reported characteristics of care utilization}

The study evaluated the medical contacts between patients and PCPs, differentiating between general medical concerns and medical questions specifically related to the underlying CHD. For primarily non-cardiac medical issues, $87.9 \%(n=3,524)$ of the ACHD predominantly consulted a PCP and only $7.2 \%(n=287)$ predominantly consulted a cardiologist. In the case of medical questions directly related to the underlying $\mathrm{CHD}$, for their primary consult, $1,991(49.7 \%)$ study participants also consulted their PCP, $1,489(37.7 \%)$ a cardiologist, and $528(13.2 \%)$ another physician. Overall, 1,982 (53.8\%) patients consulted the same PCP for both cardiac and non-cardiac health-related issues, and 3,617 (90.2\%) participants stated that their treating physicians were informed about the existence of the CHD.

Further questions addressed the referral patterns of the attending PCPs as perceived by the included ACHD. At least 2,269 (56.6\%) had never been referred to an ACHD specialist or a specialized institution. Even in the case of cardiac problems, only 1,161 (29.0\%) of the surveyed ACHD were referred to an ACHD-specialized institution. Another 221 (5.5\%) received a referral from their PCP to an ACHD-specialized institution in order to address complaints that may have been influenced by the underlying CHD.

\section{Patient knowledge and awareness of specialized medical care structures for $A C H D$}

Another important part of the study was to determine the awareness of specific medical care structures for ACHD (Table 4). Nearly half of the respondents $(1,840 ; 45.9 \%)$ were not aware of any ACHD-specific medical services. In addition, more than half of the surveyed ACHD $(2,208 ; 55.1 \%)$ stated that they did not receive sufficient information about such supply structures.

Since patient organizations can provide important support for chronically ill ACHD, the ACHD were also asked about their knowledge in this regard. Only 1,213 (30.3\%) participants were informed about ACHD patient organizations, whereas 2,552 (63.7\%) were not familiar with such associations. 
Table 4 Knowledge and awareness of specific care structures for adults with congenital heart disease

$\mathrm{n}(\%)$

Patients' knowledge about ACHD-specific care structures (multiple answers possible)

Aware of ACHD-certificated pediatric cardiologists in private practice

$969(24.2)$

Aware of ACHD-certificated cardiologists in private practice

$771(19.2)$

Aware of certified hospitals or centers for ACHD

$1,044(26.0)$

No awareness of certified hospitals or centers for ACHD

$1,840(45.9)$

Satisfaction with level of knowledge on ACHD-specific care facilities

Sufficient information obtained

$1,213(30.3)$

Insufficient information obtained

$2,208(55.1)$

"I don't know"

$587(14.6)$

$\mathrm{ACHD}$, adults with congenital heart disease; $n$, absolute number.

\section{Need for medical advice and satisfaction with currently available ACHD resources}

Since the individual needs of the affected patients in different life situations are of great importance in the follow-up of ACHD, their need for counseling was specifically queried. The following issues were indicated by the respondents as ones for which they needed counselling: exercise capacity $(\mathrm{n}=1,377,34.4 \%)$, daily life activities $(\mathrm{n}=1,281,32.0 \%)$, rehabilitation $(\mathrm{n}=1,105,27.6 \%)$, types of education $(\mathrm{n}=865,21.6 \%)$, occupational skills $(\mathrm{n}=844$; $21.1 \%)$, genetic counselling ( $\mathrm{n}=796,19.9 \%$ ), driver's license $(\mathrm{n}=715,17.8 \%)$, and aerobic capacity $(\mathrm{n}=681,17.0 \%)$. Concerning pregnancy, 1,196 (29.8\%) of the total cohort stated a need for medical advice. The counselling demand by women was age-dependent: $49.1 \%$ of those between 18-20 years, $58.2 \%$ of those between 20-30 years, and $51.1 \%$ of those between $30-40$ years. In addition, more detailed information and advice was requested on health insurance by $1,178(29.4 \%)$ respondents, on life insurance by $1,144(28.5 \%)$, on retirement issues by 1,260 (31.4\%), and on disability certificates by $869(21.7 \%)$.

Despite all deficiencies, the surveyed ACHD were for the most part satisfied with their general medical care and even with the CHD-specific care. Although 2,208 (55.1\%) of the ACHD were not sufficiently informed about the existing structures, $3,134(78.2 \%)$ rated the current care situation as either "very good" or "good".

\section{Discussion}

Worldwide, ACHD pose a challenge to healthcare providers who care for this patient population. The number of ACHD is continuously rising, a trend that is expected to continue beyond the second half of this century. Almost all of them are chronically ill due to residua, sequelae, and complicating comorbidities. To achieve an adequate quality of life and long-term survival, many of them need proper medical care throughout their lifetime.

While children with CHD are usually competently guided by pediatric cardiologists for years, many CHD patients have to switch in early adulthood to regular adult cardiologists, who are often not specifically trained in CHD $(24,25,29)$. At this time, there is a considerable loss of follow-up, partly because patients are not interested in constantly dealing with their chronic disease, or feel that they are not understood by their surrounding and treating physicians, and partly because some patients refuse further medical or surgical interventions (30-32).

This is a worldwide problem that poses a challenge to all healthcare providers. Despite all efforts, the care of ACHD is still dissatisfying, as indicated by recent data from the accredited ACHD centers in Germany, which are capable of ensuring competent, holistic care for ACHD. Although appropriate care can only be provided by experienced ACHD cardiologists, only 22,000 out of the estimated 330,000 ACHD living in Germany are under ACHDaccredited cardiological follow-up provided in an accredited ACHD center $(33,34)$. The number of ACHDs in the care of certified cardiologists is not available, but from the personal experience of the authors, it is also likely to be small.

The current study is the first to assess, from the patients' 
perspective, health care under real-world conditions in a large CHD population: 4,008 ACHD in Germany. The study sought to determine (I) how well care-structures are known and used by ACHD, (II) which medical counselling needs ACHD have, and (III) whether ACHD are satisfied with their perceived health care.

The large number of ACHD included in the current study illustrates that, due to recent advances in modern medicine, today even patients with severe CHD, who were considered to have no realistic chance until a few decades ago, survive into adulthood. The patient-reported outcome comprises data on almost all types of CHD, from the more common and simple (e.g., septal defects, congenital valve anomalies, aortic coarctation, or tetralogy of Fallot) to very complex CHD (e.g., native or operated transposition of the great arteries, univentricular heart, or hypoplastic left heart syndrome). The cohort also included all age groups, ranging from 18 to 97 years (median 38 years).

\section{The role of cardiac and non-cardiac comorbidities in ACHD}

A recent single-center study of 800 consecutive ACHD from our institution showed that $95.6 \%$ of ACHD had relevant non-cardiac comorbidities (19). Singh et al. described an even higher number within their nationwide health record analysis of 255,355 ACHD in the United States (22). These recently published data suggest that many ACHD are not sufficiently aware of and informed about their health condition and its consequences. The current study data also confirm the importance of cardiac and noncardiac comorbidities, which were present in 60\% (Table 3) $(\mathrm{n}=2,408)$ of ACHD, including heart failure, cardiac arrhythmias, pulmonary (arterial) hypertension, other organ disorders, and mental or intellectual impairment.

\section{Current medical care for ACHD}

Unfortunately, many ACHD are unaware that they suffer from a chronic disease, and both patients and their PCPs lack awareness of the importance of lifelong follow-up for CHD, not only in severe, but also in mild or moderate, cases $(24,35,36)$. Based on the analyzed data, it is obvious that PCPs are the first contact person for ACHD for general medical questions, regardless of the patient's age, sex, or type or severity of the CHD. Moreover, PCPs are also the first advisors if medical problems related to the patients' CHD arise. This is in accordance with data from a recently published study of 767 PCPs in Germany, conducted by our center. We found that more than $83 \%$ of the surveyed PCPs cared for ACHD, but for most of them (75\%), ACHD accounts for less than $1 \%$ of their entire patient population (28).

Importantly, ACHD do not tend to visit their PCP for routine check-ups and before any medical problems arise. This is important, since many ACHD often do not correctly perceive their health status and newly emerging disease complications. This is a decisive issue, as the PCP is the main contact person who should sharpen CHD patients' awareness and emphasize the importance of regular followup visits with a congenital cardiologist. Indeed, the current data reveal that ACHD primarily consulted their PCP for non-cardiac medical issues $(87.9 \% ; n=3,524)$, which may aggravate the natural course of the patients' CHD, as well as for medical questions directly related to the underlying CHD (49.7\%; $n=1,991)$. The PCPs are usually (90.2\%; $\mathrm{n}=3,617)$ informed about the existing CHD of their patients. However, since ACHD account for only a very small proportion of their patient population, many PCPs are not fully aware of the special needs and treatment requirements of ACHD and lack clinical experience with the long-term problems of this vulnerable patient group (28).

Difficult to understand are the referral habits of the PCPs as perceived by the ACHD who participated in this study. Even in the case of cardiac problems, the surveyed ACHD were only exceptionally referred to an ACHDspecialized institution $(29.0 \% ; \mathrm{n}=1,161)$ and at least $56.6 \%$ $(n=2,269)$ had never been referred to an ACHD specialist or a specialized institution. In fact, recent data show that less than $25 \%$ of PCPs in Germany involve ACHD-specialized physicians in their ACHD treatment (28). When PCPs do refer their ACHD for further cardiological care, they usually refer them to general cardiologists, who are for the most part not specifically trained to treat ACHD. This is far from ideal. Considering the broad variety of CHD and its associated conditions, the different natural course of each CHD case, and the large number of treatment options, it is impossible to determine an adequate cardiac status and treatment regime for an adult with CHD without being specifically trained in ACHD care (37). PCPs and general cardiologists must bear in mind that the cardiac and noncardiac problems in ACHD may differ significantly from those in patients with acquired heart disease. The diagnosis and management of these medical problems, as well as their 
early and correct detection, is essential (38). Therefore, ACHD strongly benefit from specialized management, which reduces morbidity and mortality (36).

\section{Knowledge and awareness of specialized resources for ACHD}

Nearly half of the respondents $(n=1,840 ; 45.9 \%)$ were unaware of the nationwide available ACHD-specific care facilities. In addition, only $30 \%(n=1,213)$ of the surveyed ACHD ( $\mathrm{n}=2,208 ; 55.1 \%)$ felt sufficiently informed about the existing ACHD-specific care facilities. Moreover, only a minority ( $\mathrm{n}=1,213,32.2 \%)$ of the questioned ACHD were familiar with ACHD patient organizations, which can often provide important support and valuable information for chronically ill ACHD and their health care needs.

\section{Counselling needs among ACHD, and future aspects}

A particular challenge in ACHD care is the tremendous demand among ACHD for advice that goes beyond the purely medical scope. When inquired in detail, the majority of ACHD stated major consultation needs concerning exercise capacity and daily life activities, pregnancy (age-dependent) and genetics, education and occupational skills, rehabilitation, and/or insurance issues. To advise ACHD on these issues, health care professionals require a great deal of expertise and experience going far beyond the competence of a generally trained PCP or general cardiologist. With respect to these issues in particular, the support of patient organizations can be extremely beneficial.

Considering the high demand for medical advice with regard to pregnancy, as an example, it is strongly recommended to advise ACHD early about their options and medical or genetic risks, including regarding contraindications and appropriate and effective contraception. Because of pregnancy-related hemodynamic changes, interdisciplinary care must be offered and organized in advance. Particularly in such cases, PCPs must urgently fulfil their responsibilities and refer all ACHDs to specialized ACHD cardiologists, regardless of the severity of the diagnosis and in spite of a subjectively good state of health $(11,39,40)$.

Despite all deficiencies, ACHD were for the most part satisfied with their general medical- and CHD-specific care. Although this finding may seem contradictory at first sight, most patients report a moderate degree of disability in their daily lives and may therefore not necessitate ACHD specialized care for a long period of time. From a life-stage perspective, their supply deficit often only becomes evident, when they eventually develop symptoms and disruptions later in life $(31,41)$.

\section{Changes resulting from the current study data}

As an immediate response to our present study, educational campaigns have been launched nationwide on television, the general press, and the Internet, supported by the German Heart Societies, patient organizations (e.g., German Heart Foundation, Herzkind e.V.), and even with the support of the pharmaceutical industry. In addition, other medical specialties, including general medicine, internal medicine, intensive care medicine, rehabilitation medicine, gynecology and obstetrics, genetics, psychology and psychiatry, sociology, nutrition, and sports medicine, are increasingly obtaining information at conferences and congresses in the context of scientific seminars.

\section{Future aspects of medical care for ACHD}

In the future, modern health care for ACHD will require better education and increased awareness among PCPs, ACHD health care providers, insurance companies, and within the ACHD patient population itself about ACHDspecific problems and special needs. Health care for ACHD should encompass patient education as part of their disease management, individually adapted to the patients' medical counselling needs. There is increasing evidence that many ACHD need support concerning psychological difficulties, dietary patterns, and exercise capacity in their daily life $(35,42,43)$. Therefore, it is of particular importance to individually advise ACHD on these demanding issues in order to prevent a high proportion of comorbidities that could be caused by unhealthy lifestyle habits, and to give ACHD the chance to take more responsibility in their own lives (44).

For all these purposes, the support of the nationwide available patient organizations is critical.

\section{Study limitations}

The data collected are based exclusively on patient-reported outcomes. Therefore, no validation of the diagnosis with respect to comorbidities was carried out. It is also not 
possible to say why some patients chose to fill out the study questionnaire while others rejected it. In addition, patients were permitted to utilize help from another person in completion of the questionnaire (partially or entirely), and this may have introduced bias into the data.

A selection bias should be considered, as most of the questionnaires were handed out to ACHD undergoing treatment at a tertiary care center. Hence, it can be assumed that the study collective differs from the general ACHD collective, concerning severity and overall awareness of lifelong necessary follow-up. It can be assumed that ACHD who are in continuous follow-up in a tertiary care center are probably more actively interested in ACHD topics, and these patients were more prone to participate in the study. Therefore, it is possible that, in reality, ACHD without specific follow-up are even less aware of CHD-related issues and existing ACHD resources. As a result, those ACHD for whom the lost-to follow-up problem is particularly relevant are probably underrepresented in the present collective survey.

Finally, the generalization of the conclusions to the primary care of ACHD in other countries is only possible to a limited extent.

\section{Conclusions}

In the future, specialized ACHD care has to consider all available treatment options, individually adapted to patients' needs and provided by specially trained ACHD health care professionals.

The results of the present study showed that many ACHD are not sufficiently aware of and informed about their health condition and its consequences, particularly regarding the need for lifelong follow-up. Many are also not sufficiently informed and/or unaware of existing ACHD health care facilities, despite the high rate of cardiac and non-cardiac co-morbidities and the immense amount of medical counselling needs among ACHD.

In addition to patient awareness, awareness of PCPs must be increased regarding the specific needs of ACHD, so that all contemporary treatment options are provided to improve the quality of life of ACHD and to prevent further disease burdens. A timely referral to a congenital cardiologist or a specialized ACHD facility may help to prevent or delay major complications in the natural course of the disease.

Finally, the presented data can be exemplary for the development of corresponding ACHD structures in other countries.

\section{Acknowledgments}

The authors would like to thank the German Heart Foundation ("Deutsche Herzstiftung e.V."), the patient organization "Herzkind e.V.", and the German health care insurance AOK-Bayern for the promotion of ACHD research or co-funding the survey. This study was also funded as an investigator-initiated study by Actelion Pharmaceuticals, Germany, GmbH. Further, the authors would like to thank the Competence Network for Congenital Heart Defects, which has received funding from the Federal Ministry of Education and Research, grant number 01GI0601 (until 2014), and the DZHK (German Centre for Cardiovascular Research, as of 2015) for the support. We explicitly thank Dr. Claudia S. Copeland for the professional editing of the final draft of the manuscript. Funding: This research was funded by the German Heart Foundation ("Deutsche Herzstiftung e.V."), grant number F-30-15, the patient organization "Herzkind e.V." and Actelion Pharmaceuticals Germany GmbH, grant number MED-2015-495.

\section{Footnote}

Provenance and Peer Review: This article was commissioned by the editorial office, Cardiovascular Diagnosis and Therapy for the series "Current Management Aspects in Adult Congenital Heart Disease (ACHD): Part III". The article has undergone external peer review.

Reporting Checklist: The authors have completed the SURGE reporting checklist. Available at http://dx.doi. org/10.21037/cdt-20-825

Data Sharing Statement: Available at http://dx.doi. org/10.21037/cdt-20-825

Peer Review File: Available at http://dx.doi.org/10.21037/ cdt-20-825

Conflicts of Interest: All authors have completed the ICMJE uniform disclosure form (available at http:// dx.doi.org/10.21037/cdt-20-825). The series "Current Management Aspects in Adult Congenital Heart Disease (ACHD): Part III" was commissioned by the editorial 
office without any funding or sponsorship. HB serves as an unpaid editorial board member of Cardiovascular Diagnosis and Therapy from Feb 2018 to Jan 2022 and served as the unpaid Guest Editor of the series. The authors have no other conflicts of interest to declare.

Ethical Statement: The authors are accountable for all aspects of the work in ensuring that questions related to the accuracy or integrity of any part of the work are appropriately investigated and resolved. The study was conducted in accordance with the Declaration of Helsinki (as revised in 2013). The study employed a questionnaire-based survey that was initiated by the Department of Congenital Heart Disease of the German Heart Center Munich, Technical University Munich. Inclusion criteria included the presence of a confirmed CHD, a minimum age of 18 years, and a written declaration of consent. Exclusion criteria were lack of cognitive competence to consent to research and refusal to consent. All data collection was conducted with the master-approval of the Ethics Committee of the Technical University of Munich, conferred on April 5, 2017 (157/16 S).

Open Access Statement: This is an Open Access article distributed in accordance with the Creative Commons Attribution-NonCommercial-NoDerivs 4.0 International License (CC BY-NC-ND 4.0), which permits the noncommercial replication and distribution of the article with the strict proviso that no changes or edits are made and the original work is properly cited (including links to both the formal publication through the relevant DOI and the license). See: https://creativecommons.org/licenses/by-nc-nd/4.0/.

\section{References}

1. EUROCAT. Cases and prevalence (per 10,000 births) of all congenital anomaly subgroups for all registries, from 2012 - 2016 08.06.2018. Available online: http://www. eurocat-network.eu/ACCESSPREVALENCEDATA/ PrevalenceTables

2. Mitchell SC, Korones SB, Berendes HW. Congenital heart disease in 56,109 births. Incidence and natural history. Circulation 1971;43:323-32.

3. Ávila P, Mercier LA, Dore A, et al. Adult congenital heart disease: a growing epidemic. Can J Cardiol 2014;30:S410-9.

4. Wren C, O'Sullivan JJ. Survival with congenital heart disease and need for follow up in adult life. Heart
2001;85:438-43.

5. Schwedler G, Lindinger A, Lange PE, et al. Frequency and spectrum of congenital heart defects among live births in Germany: a study of the Competence Network for Congenital Heart Defects. Clin Res Cardiol 2011;100:1111-7.

6. Kaemmerer H, Hess J. Adult patients with congenital heart abnormalities: present and future. Dtsch Med Wochenschr 2005;130:97-101.

7. Hoffman JI. The global burden of congenital heart disease. Cardiovasc J Afr 2013;24:141-5.

8. van der Linde D, Konings EE, Slager MA, et al. Birth prevalence of congenital heart disease worldwide: a systematic review and meta-analysis. J Am Coll Cardiol 2011;58:2241-7.

9. Drenthen W, Boersma E, Balci A, et al. Predictors of pregnancy complications in women with congenital heart disease. Eur Heart J 2010;31:2124-32.

10. Hauser M, Lummert E, Braun SL, et al. Nichtkardiale Komorbiditäten bei erwachsenen Patienten mit angeborenen Herzfehlern. Zeitschrift für Herz-, Thoraxund Gefäßchirurgie 2017;31:130-7.

11. Schmaltz AA, Bauer UM. Adults with congenital heart disease: treatment and medical problems. Herz 2013;38:639-51; quiz 52-4.

12. Baumgartner $\mathrm{H}$. An important attempt to improve the outcome of congenital heart disease in Europe. Eur Heart J 2014;35:674-5.

13. Webb G, Mulder BJ, Aboulhosn J, et al. The care of adults with congenital heart disease across the globe: Current assessment and future perspective: A position statement from the International Society for Adult Congenital Heart Disease (ISACHD). Int J Cardiol 2015;195:326-33.

14. Perloff JK, Warnes CA. Challenges posed by adults with repaired congenital heart disease. Circulation 2001;103:2637-43.

15. Warnes CA. The adult with congenital heart disease: born to be bad? J Am Coll Cardiol 2005;46:1-8.

16. Baumgartner H, De Backer J, Babu-Narayan SV, et al. 2020 ESC Guidelines for the management of adult congenital heart disease. Eur Heart J 2021;42:563-645.

17. Ministeri M, Alonso-Gonzalez R, Swan L, et al. Common long-term complications of adult congenital heart disease: avoid falling in a H.E.A.P. Expert Rev Cardiovasc Ther 2016;14:445-62.

18. Kaemmerer AS, Freilinger S, Andonian C, et al. Provision of medical health care for adults with congenital heart 
disease associated with aortic involvement. Cardiovasc Diagn Ther 2021;11:518-28.

19. Neidenbach RC, Lummert E, Vigl M, et al. Non-cardiac comorbidities in adults with inherited and congenital heart disease: report from a single center experience of more than 800 consecutive patients. Cardiovasc Diagn Ther 2018;8:423-31.

20. Andonian C, Beckmann J, Ewert P, et al. Assessment of the Psychological Situation in Adults with Congenital Heart Disease. J Clin Med 2020;9:779.

21. Lui GK, Saidi A, Bhatt AB, et al. Diagnosis and Management of Noncardiac Complications in Adults With Congenital Heart Disease: A Scientific Statement From the American Heart Association. Circulation 2017;136:e348-92.

22. Singh S, Desai R, Fong HK, et al. Extra-cardiac comorbidities or complications in adults with congenital heart disease: a nationwide inpatient experience in the United States. Cardiovasc Diagn Ther 2018;8:814-9.

23. Hess J, Bauer U, de Haan F, et al. Recommendations for adult and paediatric cardiologists on obtaining additional qualification in "Adults with Congenital Heart Disease" (ACHD). Int J Cardiol 2011;149:186-91.

24. Kaemmerer H, Bauer U, de Haan F, et al. Recommendations for improving the quality of the interdisciplinary medical care of grown-ups with congenital heart disease (GUCH). Int J Cardiol 2011;150:59-64.

25. Schmaltz AA, Bauer U, Baumgartner H, et al. Medical guideline for the treatment of adults with congenital heart abnormalities of the German-Austrian-Swiss Cardiology Specialty Society. Clin Res Cardiol 2008;97:194-214.

26. Kaemmerer H, Breithardt G. Recommendations for the quality improvement of interdisciplinary care of adults with congenital heart anomalies. Clin Res Cardiol 2006;95 Suppl 4:76-84.

27. Hess J, Bauer U, de Haan F, et al. Empfehlungen für Erwachsenen- und Kinderkardiologen zum Erwerb der Zusatz-Qualifikation „Erwachsene mit angeborenen Herzfehlern” (EMAH). Clin Res Cardiol Supp 2007;2:19-26.

28. Seidel L, Nebel K, Achenbach S, et al. Facts about the General Medical Care of Adults with Congenital Heart Defects: Experience of a Tertiary Care Center. J Clin Med 2020;9:1943.

29. Neidenbach R, Kaemmerer H, Pieper L, et al. Striking Supply Gap in Adults with Congenital Heart Disease? Dtsch Med Wochenschr 2017;142:301-3.

30. Helm PC, Kaemmerer H, Breithardt G, et al. Transition in Patients with Congenital Heart Disease in Germany: Results of a Nationwide Patient Survey. Front Pediatr 2017;5:115.

31. Wray J, Frigiola A, Bull C. Loss to specialist follow-up in congenital heart disease; out of sight, out of mind. Heart 2013;99:485-90.

32. Goossens E, Stephani I, Hilderson D, et al. Transfer of adolescents with congenital heart disease from pediatric cardiology to adult health care: an analysis of transfer destinations. J Am Coll Cardiol 2011;57:2368-74.

33. Meinertz T, Hamm C, Schlensak C, et al. Deutscher Herzbericht 2017 29. Bericht/Sektorenübergreifende Versorgungsanalyse zur Kardiologie, Herzchirurgie und Kinderherzmedizin in Deutschland. Germany: Deutsche Herzstiftung e.V., 2018:260.

34. Deutsche Herzstiftung eV. Deutscher Herzbericht 2018 2019. Available online: https://www.herzstiftung.de/ e-paper/\#0

35. Neidenbach R, Achenbach S, Andonian C, et al. Medical care of adults with congenital heart diseases: Present and future. Herz 2019;44:553-72.

36. Mylotte D, Pilote L, Ionescu-Ittu R, et al. Specialized adult congenital heart disease care: the impact of policy on mortality. Circulation 2014;129:1804-12.

37. Cohen SB, Ginde S, Bartz PJ, et al. Extracardiac complications in adults with congenital heart disease. Congenit Heart Dis 2013;8:370-80.

38. Budts W, Roos-Hesselink J, Radle-Hurst T, et al. Treatment of heart failure in adult congenital heart disease: a position paper of the Working Group of GrownUp Congenital Heart Disease and the Heart Failure Association of the European Society of Cardiology. Eur Heart J 2016;37:1419-27.

39. Vigl M, Kaemmerer M, Seifert-Klauss V, et al. Contraception in women with congenital heart disease. Am J Cardiol 2010;106:1317-21.

40. Uebing A, Gatzoulis MA, von Kaisenberg C, et al. Congenital heart disease in pregnancy. Dtsch Arztebl Int 2008;105:347-54.

41. Gurvitz M, Marelli A, Mangione-Smith R, et al. Building quality indicators to improve care for adults with congenital heart disease. J Am Coll Cardiol 2013;62:2244-53.

42. Andonian C, Beckmann J, Biber S, et al. Current research status on the psychological situation of adults with congenital heart disease. Cardiovasc Diagn Ther 2018;8:799-804.

43. Andonian C, Langer F, Beckmann J, et al. Overweight and Obesity: An Emerging Problem in Patients with 
Congenital Heart Disease submitted 2019. Cardiovasc Diagn Ther 2019;9:S360-8.

44. Moons P, Hilderson D, Van Deyk K. Implementation of

Cite this article as: Neidenbach R, Achenbach S, Andonian C, Bauer UMM, Ewert P, Freilinger S, Gundlach U, Kaemmerer AS, Nagdyman N, Nebel K, Oberhoffer R, Pieper L, Reinhard W, Sanftenberg L, von Scheidt F, Schelling J, Seidel L, Weyand M, Kaemmerer H. Systematic assessment of health care perception in adults with congenital heart disease in Germany. Cardiovasc Diagn Ther 2021;11(2):481-491. doi: 10.21037/cdt-20-825 transition programs can prevent another lost generation of patients with congenital heart disease. Eur J Cardiovasc Nurs 2008;7:259-63. 